\title{
The Multipliers of Periodic Points in One-dimensional Dynamics
}

\author{
Marco Martens ${ }^{1}$ Welington de Melo $^{2}$ \\ September 30,1997
}

\begin{abstract}
.
It will be shown that the smooth conjugacy class of an $S$-unimodal map which does not have a periodic attractor neither a Cantor attractor is determined by the multipliers of the periodic orbits. This generalizes a result by M.Shub and D.Sullivan for smooth expanding maps of the circle.
\end{abstract}

${ }^{1}$ Institute for Mathematical Sciences, SUNY at Stony Brook, Stony Brook, NY 11794-3651.

${ }^{2}$ IMPA, Estrada Dona Castorina 110, 22460-320, Rio de Janeiro, Brazil. Partially supported by Pronex project on Dynamical Systems 


\section{Introduction}

Let $f$ and $g$ be topologically equivalent smooth interval maps. That means, there is a homeomorphism $h$ such that $h \circ f=g \circ h$. We will say that $f$ and $g$ have the same multipliers if for every periodic point $x$ with period $n$

$$
D g^{n}(h(x))=D f^{n}(x) .
$$

Let $\mathcal{U}$ be the set of unimodal maps $f:[-1,1] \rightarrow[-1,1]$ of the form

$$
f=\phi \circ q_{t},
$$

where $\phi:[-1,1] \rightarrow[-1,1]$ is an orientation preserving $C^{3}$ diffeomorphism with negative Schwarzian derivative and $q_{t}:[-1,1] \rightarrow[-1,1]$ a canonical folding map

$$
q_{t}(x)=-2 t|x|^{\alpha}+2 t-1
$$

for some $t \in[0,1]$ and $\alpha>1$. The number $\alpha$ is called the critical exponent of $f$. The maps in $\mathcal{U}$ are often called $S$-unimodal maps.

It has been shown in $[\mathrm{BL}],[\mathrm{Ma}]$ that for every map $f \in \mathcal{U}$ there exists a unique closed invariant set $A_{f} \subset[-1,1]$ such that

$$
\omega(x)=A_{f}
$$

for almost every $x \in[-1,1]$ (in Lebesgue sense). Here $\omega(x)$ denotes the limit set of $x$. This set $A_{f}$ is the attractor of $f$ as defined by Milnor [Mi].

There are three possibilities: the attractor is a periodic orbit, or it is a Cantor set, or it is the orbit of a periodic interval. This work will concentrate on maps whose attractor is of the third type.

$$
\mathcal{U}_{0}=\left\{f \in \mathcal{U} \mid \text { the topological dimension of } A_{f} \neq 0\right\} .
$$

Theorem. Let $f, g \in \mathcal{U}_{0}$ be conjugated by a homeomorphism $h$. If $f$ and $g$ have the same multipliers then they belong to the same $C^{2}$ conjugacy class, that is, $h: A_{f} \rightarrow A_{g}$ is a $C^{2}$ diffeomorphism.

This Theorem is generalization of a result obtained by M.Shub and D.Sullivan. They proved that two smooth expanding circle maps with the same multipliers are smoothly conjugated [SS]. In [L1] it has been shown that $C^{2}$ unimodal maps with Fibonnaci combinatorics and with the same multipliers are $C^{1}$ conjugated. The proof of the Theorem will be by joining the methods in [SS] and [Ma].

For $S$-unimodal maps with critical exponent $\alpha=2$ it has been proved in [L2] that a Cantor attractor only appears for infinitely renormalizable maps (see also [LM]). Hence for such unimodal maps the multipliers of the periodic orbits form a complete smooth invariant whenever the map does not have a periodic attractor and only finitely many renormalization. In [BKNS] examples have been constructed of non-renormalizable unimodal maps, with high critical exponent, exhibiting Cantor attractors. A characterization of the smooth conjugacy classes of such maps is not known. 
Conjecture. Let $f, g \in \mathcal{U}$ be unimodal maps which do not have periodic attractors neither Cantor attractors. If $f$ and $g$ are $C^{r}, r \geq 2$, and have the same multipliers then they are conjugated by a $C^{r}$ diffeomorphism.

In the context of unimodal maps which have a quadratic-like extension, the method presented here will prove that having the same multipliers implies that the conjugation is even real analytic. The proof of Lemma 2.6 and 3.3 will have to be changed slightly by using the Koebe-Lemma for univalent maps.

An appendix is added in which some basic notions are defined.

\section{Acknowledgements}

The first author would like to thank IMPA for its kind hospitality during august 1997, the period in which this rather serendipitous result was obtained.

\section{Markov-Maps}

In section 3. the conjugacy problem between unimodal maps will be reduced to the conjugacy problem between Markov-maps. Before we define Markov-maps we will discuss the Banach space Diffr ${ }_{+}([-1,1]), r \geq 2$, consisting of $C^{r}$ orientation preserving diffeomorphisms of $[-1,1]$. The Banach space structure is obtained by the following identification. Let $\eta: \operatorname{Diff}_{+}^{r}([-1,1]) \rightarrow C^{r-2}([-1,1])$ defined by

$$
\eta_{\phi}=D \ln D \phi .
$$

This bijection $\eta$ is called the non-linearity. The usual Banach space structure on $C^{r-2}([-1,1])$ can be pulled back onto Diffr $+([-1,1])$. In particular

$$
|\phi|_{r}=\sum_{k \leq r-2}\left\|D^{k} \eta_{\phi}\right\|_{0},
$$

where $\|.\|_{0}$ denotes the $C^{0}$ norm on the space of continuous functions.

Furthermore, if $f: T \rightarrow f(T)$ is an orientation preserving diffeomorphism defined on the interval $T$ then

$$
[f \mid T] \in \operatorname{Diff}_{+}^{r}([-1,1])
$$

denotes the orientation preserving diffeomorphism obtained by rescaling range and domain of $f$.

Definition 2.1. A map $F: \bigcup M_{i} \rightarrow[-1,1]$ where $\left\{M_{i} \mid i \in \mathbb{N}\right\}$ is a countable collection of closed nondegenerate oriented subintervals in $[-1,1]$ with the property

$$
\left|[-1,1] \backslash \bigcup M_{i}\right|=0,
$$


is called a $C^{r}$ Markov-map, $r \geq 3$, if

1) $F: M_{i} \rightarrow[-1,1]$ is an orientation preserving diffeomorphism for every $i \in \mathbb{N}$.

2) There exists $a>0$ such that for every $i \in \mathbb{N}$ there exists an interval $[-1,1] \supset T_{i} \supset$ $M_{i}$ and an extension $\hat{F}: T_{i} \rightarrow[-1-a, 1+a]$ of $F: M_{i} \rightarrow[-1,1]$ which is a $C^{r}$ diffeomorphism with negative Schwarzian derivative.

3) There exists $K<0$ such that $\left|\left[\left(F \mid M_{i}\right)^{-1}\right]\right|_{r} \leq K$ for every $M_{i}$.

Observe, that the maps $F \mid M_{i}$ can be orientation reversing when the usual orientation on $M_{i}$ is used.

Let $H$ be a homeomorphism conjugating the $C^{r}$ Markov-maps $F$ and $G$. The conjugacy $H$ preserves the multipliers iff for every periodic point $x$ of $F$ with period $n$

$$
D G^{n}(H(x))=D F^{n}(x) .
$$

Observe that in general there are many conjugating homeomorphisms between two given Markov-maps. This is the reason why the above definition differs from the one concerning unimodal maps.

Proposition 2.2. Every conjugation between Markov-maps $F$ and $G$ which preserves the multipliers is Lipschitz. In particular, such a conjugation is absolutely continuous with respect to the Lebesgue measure.

Proposition 2.3. For every $C^{r}$ Markov-map, $r \geq 3$, there is a $C^{r}$ diffeomorphism $H$ : $[-1,1] \rightarrow[-1,1]$ such that

$$
F_{0}=H^{-1} \circ F \circ H
$$

is a $C^{r}$ Markov-map which preserves the Lebesgue measure.

The proofs needs some preparation. Let $\Sigma$ be the one-sided symbol sequence over the alphabet $\mathbb{N}$. Take $w \in \Sigma$ and consider the finite word $w_{n}$ consisting of only the first $n$ symbols of $w$. There is an unique interval $I_{w}^{n} \subset[-1,1]$ such that

1) $F^{n}: I_{w}^{n} \rightarrow[-1,1]$ is a diffeomorphism.

2) $F^{j}\left(I_{w}^{n}\right) \subset M_{w(n-j)}$ for $j=0,1, \cdots, n-1$.

The map $F^{n}: I_{w}^{n} \rightarrow[-1,1]$ is called the branch of $F^{n}$ with the combinatorics $w_{n}$. The collection of intervals $\left\{I_{w}^{n} \mid w \in \Sigma\right\}$ is denoted by $\mathcal{I}^{n}$. For every $w \in \Sigma$ and $n \geq 0$ let $\psi_{w, n}, \phi_{w, n} \in \operatorname{Diff}_{+}^{r}([-1,1])$ be defined by

$$
\psi_{w, n}=\left[\left(F^{n} \mid I_{w}^{n}\right)^{-1}\right]
$$

and

$$
\phi_{w, n}=\left[\left(F \mid I_{w}^{n+1}\right)^{-1}\right] .
$$

Observe that

$$
\psi_{w, n+1}=\phi_{w, n} \circ \psi_{w, n} .
$$

Lemma 2.4. The collections $\mathcal{I}^{n}$ have the following properties.

1) $\left|[-1,1] \backslash \bigcup_{w} I_{w}^{n}\right|=0$ for each $n \geq 0$.

2) For every $I_{w}^{n} \in \mathcal{I}^{n}$ there exists a unique interval $T_{w}^{n}$ and a unique $J \in \mathcal{I}^{n-1}$ such that 
- $I_{w}^{n} \subset T_{w}^{n} \subset J$ and

- $\hat{F}^{n}: T_{w}^{n} \rightarrow[-1-a, 1+a]$ is a diffeomorphism.

3) There is a $\delta<1$ and $C>0$ such that

$$
\frac{\left|I_{w}^{n}\right|}{\left|M_{w(n)}\right|}, \frac{\left|F\left(I_{w}^{n}\right)\right|}{\left|M_{w(n-1)}\right|} \leq C \delta^{n}
$$

In particular, the distortion of $F^{n} \mid I_{w}^{n}$ (and $\psi_{w, n}$ ) is uniformly bounded (the bound $K$ is independent of $w \in \Sigma$ and $n \geq 0$ ). Moreover, $F$ is ergodic.

Proof: Statement 1) and 2) follow directly from the definition of Markov-maps. Statement 3) and the distortion statement is then a direct consequence of the Koebe-Lemma (see the Appendix). To prove the ergodicity we have to show that any given invariant set $X$ with positive Lebesgue measure has full measure. Let $D \subset[-1,1]$ be the biggest set on which each $F^{n}$ is defined. This set $D$ has full measure. Take a density point of $X$ with $x \in X \cap D$. Then for each $n \geq 0$ there is $I_{w_{n}}^{n}$ with $x \in I_{w_{n}}^{n}$. Observe

$$
\frac{|[-1,1] \backslash X|}{|[-1,1]|} \leq \lim _{n \rightarrow \infty} \frac{\left|F^{n}\left(I_{w_{n}}^{n} \backslash X\right)\right|}{\left|F^{n}\left(I_{w_{n}}^{n}\right)\right|} \leq \lim _{n \rightarrow \infty} K \frac{\left|I_{w_{n}}^{n} \backslash X\right|}{\left|I_{w_{n}}^{n}\right|} .
$$

Here we used that the distortion of $F^{n} \mid I_{w_{n}}^{n}$ is uniformly bounded by $K$. The intervals $I_{w_{n}}^{n}$ shrink down to the density point $x$ of $X$, the last limit has to equal zero. We proved that $X$ has full measure.

I (Lemma 2.4)

As a consequence of Lemma 2.4 we see that the derivatives of $\psi_{w, n}$ are uniformly bounded by $K$. In particular, we can define for each $n \geq 0$ the density

$$
\rho_{n}(x)=\frac{1}{2} \cdot \sum_{I_{w}^{n} \in \mathcal{I}^{n}}\left|D \psi_{w, n}(x)\right| \cdot\left|I_{w}^{n}\right| .
$$

These densities are, because of Lemma 2.4, uniformly bounded and uniformly away from zero. Moreover, observe that $\rho_{n}$ is the density of $\mu_{n}=F_{*}^{n}\left(\frac{1}{2} \lambda\right)$, where $F_{*}$ be the PerronFrobenius operator and $\lambda$ is the Lebesgue measure on $[-1,1]$. Let $\mu$ be a probability measure on $[-1,1]$ which is a weak limit of the sequence

$$
\frac{1}{n} \sum_{i=0}^{n-1} \mu_{i}
$$

This measure $\mu$ is an invariant measure for $F$. The next Lemma will imply that $\mu$ is absolutely continuous. The ergodicity of $F$ implies that there is only one absolutely continuous invariant measure: the above sequence of measures is actually convergent.

Lemma 2.5. There is a continuos function $k:[0,2) \rightarrow[1, \infty)$ with $k(0)=1$ such that for every interval $T \subset[-1,1]$ and every measurable $A \subset T$

$$
\frac{1}{k(|T|)} \frac{|A|}{|T|} \leq \frac{\mu(A)}{\mu(T)} \leq k(|T|) \frac{|A|}{|T|} .
$$


Proof: The Koebe-Lemma (see Appendix) implies the existence of a continuous function $k:[0,2] \rightarrow[1, \infty)$ with $k(0)=1$, such that for every interval $T \subset[-1,1], I_{w}^{n} \in \mathcal{I}^{n}$ and $x, y \in I_{w}^{n}$ with $F^{n}(x), F^{n}(y) \in T$

$$
\frac{1}{k(|T|)} \leq \frac{D F^{n}(x)}{D F^{n}(y)} \leq k(|T|) .
$$

Hence

$$
\frac{1}{k(|T|)} \leq \frac{D \psi_{w, n}(x)}{D \psi_{w, n}(y)} \leq k(|T|)
$$

for every $x, y \in T$.

It is enough to prove the Lemma when the set $A \subset T$ is an interval. Let $A \subset T \subset[-1,1]$ be intervals. Then there exist for every $I_{w}^{n}$ points $a_{w, n} \in A$ and $t_{w, n} \in T$ such that

$$
\frac{\mu_{n}(A)}{\mu_{n}(T)}=\frac{\sum_{I_{w}^{n} \in \mathcal{I}^{n}}\left|D \psi_{w, n}\left(a_{w, n}\right)\right|}{\sum_{I_{w}^{n} \in \mathcal{I}^{n}}\left|D \psi_{w, n}\left(t_{w, n}\right)\right|} \cdot \frac{|A|}{|T|}
$$

Using the above distortion estimate we get

$$
\frac{1}{k(|T|)} \cdot \frac{|A|}{|T|} \leq \frac{\mu_{n}(A)}{\mu_{n}(T)} \leq k(|T|) \cdot \frac{|A|}{|T|} .
$$

This bound is independent of $n$. Hence it will also hold for any weak limit $\mu$ of $\frac{1}{n} \sum_{i=0}^{n-1} \mu_{i}$. The Lemma is proved.

】 (Lemma 2.5)

Lemma 2.6. The sequence $\psi_{w, n} \in \operatorname{Diff}_{+}^{r}([-1,1])$ is a Cauchy sequence for every $w \in \Sigma$. Let $\psi_{w}=\lim \psi_{w, n}$. The function $\Psi: \Sigma \rightarrow \operatorname{Diff}_{+}^{r}([-1,1])$ with

$$
\Psi(w)=\psi_{w}
$$

is continuous.

Proof: Let $w \in \Sigma$ and $n \geq 0$. Remember that $\phi_{w, n} \in \operatorname{Diff}_{+}^{r}([-1,1])$ was constructed such that

$$
\psi_{w, n+1}=\phi_{w, n} \circ \psi_{w, n}
$$

Observe that

$$
\phi_{w, n}=\left[\left(F \mid I_{w}^{n+1}\right)^{-1}\right]=\left[\left(F \mid M_{w(n+1)}\right)^{-1} \mid F\left(I_{w}^{n+1}\right)\right] .
$$

Hence, as an immediate consequence of Lemma 2.4(3) and definition 2.1(3) we get a uniform $C>0$ and $\delta<1$ with

$$
\left|\phi_{w, n}\right|_{r} \leq C \delta^{n} .
$$

Claim. There exists $K>0$ such that

$$
\left|\psi_{w, n+1}-\psi_{w, n}\right|_{r} \leq K\left|\phi_{w, n}\right|_{r}
$$


ProOF: For every $k \geq 0$ there exists polynomials $P_{j}^{k}: \mathbb{R}^{k+1} \rightarrow \mathbb{R}, j \leq k$ such that for every pair $\phi, \psi \in \operatorname{Diff}_{+}^{r}([-1,1])$ we have

$$
D^{k}\left(\eta_{\phi}(\psi(x)) D \psi(x)\right)=\sum_{j=0}^{k} D^{j} \eta_{\phi}(\psi(x)) \cdot P_{j}^{k}\left(D \psi(x), D^{2} \psi(x), \cdots, D^{k+1} \psi(x)\right),
$$

for every $x \in[-1,1]$. Using this formula we will inductively prove the Claim. By the chain rule and the definition

$$
\left|\psi_{w, n+1}-\psi_{w, n}\right|_{r}=\left|\left(\eta_{\phi_{w, n}} \circ \psi_{w, n} \cdot D \psi_{w, n}\right)\right|_{r-2}=\sum_{j=0}^{r-2}\left|D^{j}\left(\eta_{\phi_{w, n}} \circ \psi_{w, n} \cdot D \psi_{w, n}\right)\right|_{0} .
$$

First we will prove the Claim for $r=2$. Observe

$$
\begin{aligned}
\left|\psi_{w, n+1}-\psi_{w, n}\right|_{2} & =\left|\eta_{\phi_{w, n}} \circ \psi_{w, n} \cdot D \psi_{w, n}\right|_{0} \\
& \leq K\left|\eta_{\phi_{w, n}}\right|_{0} .
\end{aligned}
$$

We used Lemma 2.4: the maps $\psi_{w, n}$ have uniformly bounded derivative.

Assume the Claim is proved for $r \geq 2$ (to do the induction step we have to assume that the Markov-map is at least $\left.C^{r+1}\right)$. The induction assumption implies that the sequence $\psi_{w, n}$ is a Cauchy sequence: $\psi_{w, n} \rightarrow \psi_{w} \in \operatorname{Diff}_{+}^{r}([-1,1])$. In particular

$$
D^{k} \psi_{w, n} \rightarrow D^{k} \psi_{w}, k \leq r
$$

in the $C^{0}$ topology. Now use this convergence and above the expression for derivatives $D^{k}\left(\eta_{\phi}(\psi(x)) D \psi(x)\right)$ to get a bound

$$
D^{(r+1)-2}\left(\eta_{\phi_{w, n}}\left(\psi_{w, n}(x)\right) D \psi_{w, n}(x)\right) \leq K \sum_{s \leq(r+1)-2}\left|D^{s} \eta_{\phi_{w, n}}\right|_{0}=K\left|\phi_{w, n}\right|_{r+1} .
$$

The constant $K$ depends on the coefficients of the polynomials $P_{j}^{(r+1)-2}$ and the norms of the derivatives of $\psi_{w, n}$.

The main consequence of the Claim is that every sequence $\psi_{w, n}$ is a Cauchy sequence in the $C^{r}$ topology. Moreover, the constants obtained in the induction steps depend only on the initial distortion bound obtained from Lemma 2.4 and the exponential decay of $\left|\phi_{w, n}\right|_{r}$, which is also uniform. In particular, there is a uniform constant $K>0$ such that for every pair $w^{\prime}, w \in \Sigma$ with $w_{n}^{\prime}=w_{n}$ for some $n \geq 0$ we have

$$
\left|\psi_{w^{\prime}}-\psi_{w}\right|_{r} \leq K \delta^{n}
$$

The function $\Psi$ is continuous.

- (Lemma 2.6) 
The Borel $\sigma$-algebra on $\Sigma$ is generated by the cylinders:

$$
[w]_{n}=\{v \in \Sigma \mid v(j)=w(j), j \leq n\}
$$

The collection of cylinders is denoted by $\mathcal{C}$. Define the function $\nu: \mathcal{C} \rightarrow[0,1]$ by

$$
\nu\left([w]_{n}\right)=\mu\left(I_{w}^{n}\right)
$$

Lemma 2.7. The function $\nu$ extends to a measure on the Borel $\sigma$-algebra of $\Sigma$.

ProOF: Observe $[w]_{n}=\bigcup_{i}\left[w_{n} i^{\infty}\right]_{n+1}$. To prove that $\nu$ can be extended to a Borel measure it is enough to show that $\nu\left([w]_{n}\right)=\sum_{i} \nu\left(\left[w_{n} i^{\infty}\right]_{n+1}\right)$. The measure $\mu$ is invariant under $F$. Hence

$$
\nu\left([w]_{n}\right)=\mu\left(I_{w}^{n}\right)=\mu\left(F^{-1}\left(I_{w}^{n}\right)\right)=\mu\left(\bigcup_{i} I_{w_{n} i \infty}^{n+1}\right)=\sum_{i} \mu\left(I_{w_{n} i \infty}^{n+1}\right)=\sum_{i} \nu\left(\left[w_{n} i^{\infty}\right]_{n+1}\right) .
$$

- (Lemma 2.7)

Because $\Psi: \Sigma \rightarrow \operatorname{Diff}_{+}^{r}([-1,1])$ is continuous the function $\rho:[-1,1] \rightarrow(0, \infty)$ defined by

$$
\rho(x)=\int\left|D \psi_{w}(x)\right| d \nu
$$

is $C^{r-1}$. Now we get the rather peculiar

LEMma 2.8. The $C^{r-1}$ function $\rho$ is the density of the invariant measure $\mu$ of $F$.

Proof: The ergodicity of $F$ implies that it is enough to show that $\rho$ is the density of some absolutely continuous invariant measure: it has to be the density of $\mu$. First observe

$$
\rho(x)=\int\left|D \psi_{w}(x)\right| d \nu=\lim _{n \rightarrow \infty} \sum_{I_{w, n} \in \mathcal{I}^{n}}\left|D \psi_{w, n}(x)\right| \cdot \mu\left(I_{w}^{n}\right) .
$$

Denote the Perron-Frobenius operator acting on the space of densities also by $F_{*}$. Let $x \in[-1,1]$ and let $y_{i} \in I_{i}^{1}$ be such that $F\left(y_{i}\right)=x$. Then

$$
\begin{aligned}
F_{*}(\rho)(x) & =\sum_{i} \frac{1}{\left|D F\left(y_{i}\right)\right|} \cdot \rho\left(y_{i}\right) \\
& =\sum_{i} \frac{1}{\left|D F\left(y_{i}\right)\right|} \int\left|D \psi_{w}\left(y_{i}\right)\right| d \nu \\
& =\lim _{n \rightarrow \infty} \sum_{i} \sum_{I_{w}^{n} \in \mathcal{I}^{n}} \frac{1}{\left|D F\left(y_{i}\right)\right|} \cdot\left|D \psi_{w}^{n}\left(y_{i}\right)\right| \cdot \mu\left(I_{w}^{n}\right) \\
& =\lim _{n \rightarrow \infty} \sum_{i} \sum_{I_{w}^{n} \in \mathcal{I}^{n}}\left|D \psi_{i w, n+1}(x)\right| \cdot \frac{\left|I_{i w}^{n+1}\right|}{\left|I_{w}^{n}\right|} \cdot \mu\left(I_{w}^{n}\right),
\end{aligned}
$$


where $i w$ is the word obtained by concatenating $w$ after $i$. Now we will use Lemma 2.5:

$$
\begin{aligned}
F_{*}(\rho)(x) & =\lim _{n \rightarrow \infty} \sum_{i} \sum_{I_{w}^{n} \in \mathcal{I}^{n}}\left|D \psi_{i w, n+1}(x)\right| \cdot \mu\left(I_{i w}^{n+1}\right) \cdot \frac{\mu\left(I_{w}^{n}\right)}{\mu\left(I_{i w}^{n+1}\right)} \cdot \frac{\left|I_{i w}^{n+1}\right|}{\left|I_{w}^{n}\right|} \\
& =\lim _{n \rightarrow \infty} \sum_{i} \sum_{I_{w}^{n} \in \mathcal{I}^{n}}\left|D \psi_{i w, n+1}(x)\right| \cdot \mu\left(I_{i w}^{n+1}\right) \\
& =\lim _{n \rightarrow \infty} \sum_{I_{v}^{n+1} \in \mathcal{I}^{n+1}}\left|D \psi_{v, n+1}(x)\right| \cdot \mu\left(I_{v}^{n+1}\right) \\
& =\rho(x) .
\end{aligned}
$$

- (Lemma 2.8)

Proof of Proposition 2.2: Let $H$ be a multiplier preserving conjugacy between the Markov-maps $F$ and $G$. Lemma 2.4 states that each branch $F^{n}: I_{w}^{n} \rightarrow[-1,1]$ and $G^{n}: H\left(I_{w}^{n}\right) \rightarrow[-1,1]$ has uniformly bounded distortion, say bounded by $K$. Lemma $2.4(3)$ implies that each branch $F^{n}: I_{w}^{n} \rightarrow[-1,1]$ has an unique fixed point $p_{w}^{n}$. Now we can estimate the length of $I_{w}^{n}$ :

$$
\frac{1}{K} \leq \frac{2 /\left|I_{w}^{n}\right|}{\left|D F^{n}\left(p_{w}^{n}\right)\right|} \leq K
$$

And

$$
\frac{1}{K} \leq \frac{2 /\left|H\left(I_{w}^{n}\right)\right|}{\left|D G^{n}\left(H\left(p_{w}^{n}\right)\right)\right|} \leq K
$$

Because $D G^{n}\left(H\left(p_{w}^{n}\right)\right)=D F^{n}\left(p_{w}^{n}\right)$ we get

$$
\frac{1}{K} \leq \frac{\left|H\left(I_{w}^{n}\right)\right|}{\left|I_{w}^{n}\right|} \leq K
$$

for each branch $I_{w}^{n} \in \mathcal{I}^{n}$.

To prove that $H$ is Lipschitz choose $x, y \in[-1,1]$. Let

$$
\mathcal{I}_{x, y}^{n}=\left\{I \in \mathcal{I}^{n} \mid I \subset[x, y]\right\} .
$$

By using that $\left|I_{w}^{n}\right|,\left|H\left(I_{w}^{n}\right)\right| \rightarrow 0$ for $n \rightarrow \infty$ we get

$$
|H(y)-H(x)|=\lim _{n \rightarrow \infty} \sum_{I \in \mathcal{I}_{x, y}^{n}}|H(I)| \leq \lim _{n \rightarrow \infty} K \cdot \sum_{I \in \mathcal{I}_{x, y}^{n}}|I|=K|y-x| .
$$

- (Proposition 2.2)

Proof of Proposition 2.3: Let $H:[-1,1] \rightarrow[-1,1]$ be the $C^{r}$ diffeomorphism defined by

$$
H(x)=\int_{-1}^{x} \rho(t) d t .
$$

Indeed, $H$ is a diffeomorphism because $\rho$ is bounded and away from zero (see Lemma 2.5). By construction we have $H_{*}(\mu)=\frac{1}{2} \lambda$. In particular, the $C^{r}$ Markov-map $F_{0}=H^{-1} \circ F \circ H$ preserves the Lebesgue measure.

-(Proposition 2.3) 
Corollary 2.9. Every conjugacy between two $C^{r}$ Markov-maps which preserves multipliers is a $C^{r}$ diffeomorphism.

Proof: There exist $C^{r}$ Markov-maps $F_{0}$ and $G_{0}$ which preserve the Lebesgue measure and are $C^{r}$ conjugated to respectively $F$ and $G$. The homeomorphism $H$ which preserves the multipliers of $F$ and $G$ induces an exponent preserving conjugacy $H_{0}$ between $F_{0}$ and $G_{0}$. This conjugacy is absolutely continuous, by Proposition 2.2. The ergodicity of both maps $F_{0}$ and $G_{0}$, see Lemma 2.4, imply that the conjugacy $H_{0}$ has to map the invariant absolutely continuous measure of $F_{0}$ to the absolutely continuous invariant measure of $G_{0}$. However, both measure are the Lebesgue measure. Consequently, $H_{0}$ is the identity.

(Corollary 2.9)

The proof of Corollary 2.9 summarizes the arguments in [SS].

\section{Proof of the Theorem}

In this section we will prove the Theorem. Fix two non-renormalizable unimodal map $f, g \in$ $\mathcal{U}$ which does not have periodic attractors neither a Cantor attractor. Assume that they have the same exponents. In particular they are conjugated, say by the homeomorphism $h$. In this case the attractor of $f$ and $g$ will be an interval, respectively $A_{f}=\left[f^{2}(0), f(0)\right]$ and $A_{g}=\left[g^{2}(0), g(0)\right]$.

Lemma 3.1. For every interval $T \subset A_{f}$ there exist $n \geq 0$ and an interval $M \subset T$ such that $f^{n}: M \rightarrow A_{f}$ is monotone and onto.

Proof: The map $f \mid A_{f}$ is not renormalizable. Hence it is topologically conjugated to a continuous expanding piecewise affine map $f_{0}:\left[c_{2}, c_{1}\right] \rightarrow\left[c_{2}, c_{1}\right]$ with critical point 0 and $c_{2}=f^{2}(0), c_{1}=f(0)$. In [GMT] (Lemma 2.6) it has been shown that for each interval $T$ there exists an interval $M \subset T$ and $m \geq 0$ such that $f^{m}: M \rightarrow\left[c_{2}, c_{1}\right]$ is monotone and $f^{m}(M)=\left[c_{2}, 0\right]$ or $f^{m}(M)=\left[0, c_{1}\right]$. The Lemma follows immediately because $\left[f_{0}\left(c_{2}\right), f_{0}(0)\right]$ contains the expanding fixed point of $f_{0} \in\left[0, c_{1}\right]$.

(Lemma 3.1)

Take $x \in \operatorname{int}\left(A_{f}\right)$, the interior of $A_{f}$. We will describe an induced map on a neighborhood of $x$ and obtain a topologically defined $C^{2}$ Markov-map. The periodic orbits of $f$ are dense in $A_{f}$. This allows us to choose an interval $U=[a, b]$ with $x \in \operatorname{int}(U)$ and $a, b$ are periodic points whose orbit do not intersect $\operatorname{int}(U)$.

An interval $I \subset A_{f}$ is called good if there exist $n_{I}>0$ and an interval $T \supset I$ such that $f^{n_{I}}:(I, T) \rightarrow\left(U, A_{f}\right)$ is monotone and onto. Clearly, the number $n_{I}$ is uniquely defined. The interval $T$ is called the extension of $I$. As a consequence of Lemma 3.1 we get that in every open set there are good intervals.

The fact that the boundary points of $U$ do not return into int $(U)$ imply that good intervals are nested: if $I_{1}$ and $I_{2}$ are good intervals then

$$
\operatorname{int}\left(I_{1}\right) \cap \operatorname{int}\left(I_{2}\right)=\emptyset \text { or } I_{1} \subset I_{2} \text { or } I_{2} \subset I_{1} .
$$

We say that a good interval $I$ is of first generation if there does not exist a good interval $J$ which strictly contains $I$. The density of good intervals imply that also the first generation good intervals are dense. 
Let $\mathcal{I}$ be the collection of first generation good intervals which are part of $U$. Observe that there are no good intervals which intersect the boundary of $U$ in their interior. In general $\mathcal{I}$ will be a countable collection and $\overline{\bigcup \mathcal{I}}=U$. Define $M: \bigcup \mathcal{I} \rightarrow U$ by $M: I=f^{n_{I}}, I \in \mathcal{I}$.

LEMMA 3.2. $|U \backslash \bigcup \mathcal{I}|=0$.

Proof: We will use some notation from [Ma]. Let $n \geq 0$ and $x \in A_{f}$. The maximal interval on which $f^{n}$ is monotone is denoted by $T^{n}(x)$. The components of $f^{n}\left(T_{n}(x)\right)-$ $\left\{f^{n}(x)\right\}$ are denoted by $L_{n}(x)$ and $R_{n}(x)$. Define $r_{n}: A_{f} \rightarrow \mathbb{R}$ by

$$
r_{n}(x)=\min \left\{\left|L_{n}(x)\right|,\left|R_{n}(x)\right|\right\} .
$$

Furthermore define $r: A_{f} \rightarrow \mathbb{R}$ by

$$
r(x)=\limsup _{n \rightarrow \infty} r_{n}(x)
$$

It has been proved in [Ma] that there exists some $r_{f}>0$ such that

$$
r(x)=r_{f}
$$

for almost every $x \in A_{f}$. Here we used that $f$ does not have a Cantor attractor.

Now we will describe an extra condition on the periodic boundary points $a$ and $b$ of $U$. The two periodic orbits define a partition of $A_{f}: \mathcal{P}=\left\{P_{i} \mid i=1,2, \cdots, s\right\}$ is formed by the intervals between the points of the two periodic orbits. Assume that the maximal length of the intervals in this partition is smaller that $\frac{1}{10} r_{f}$. But also that the intervals in the partition $h(\mathcal{P})$ have length smaller than $\frac{1}{10} r_{g}$.

In each interval $P_{i}, i=1,2, \cdots s$ there exists a good interval $G_{i} \subset P_{i}$. The partition $\mathcal{P}$ is finite hence there exists an $\epsilon>0$ such that

$$
\frac{\left|G_{i}\right|}{\left|P_{i}\right|} \geq \epsilon, i=1,2, \cdots, s .
$$

We will prove the Lemma by contradiction. Assume that there is a density point $y \in U$ of $|U \backslash \bigcup \mathcal{I}|=0$ with $r_{f}(y)=r$. The point $y$ being a density point implies that for good intervals $I$ close to $y$

$$
\lim _{I \rightarrow y} \frac{|I|}{\operatorname{dist}(y, I)}=0 .
$$

We will construct big good intervals arbitrarily close to $y$ and obtain a contradiction to the above statement.

Because $r_{f}(y)=r$ there exists a sequence $k_{n} \rightarrow \infty$ such that $r_{k_{n}}(y) \geq \frac{1}{2} r_{f}$. For each $n \geq 0$ there exists $P_{i_{n}} \in \mathcal{P}$ such that $f^{k_{n}}(y) \in P_{i_{n}}$. Moreover, there exists an interval $M_{n} \subset T_{k_{n}}(y)$ with $y \in M_{n}$ and $f^{k_{n}}: M_{n} \rightarrow P_{i_{n}}$ monotone and onto. Because $\left|P_{i_{n}}\right| \leq \frac{1}{10} r_{f}$ and $r_{k_{n}}(y) \geq \frac{1}{2} r_{f}$ we get from the Koebe-Lemma that $f^{k_{n}}: M_{n} \rightarrow P_{i_{n}}$ has uniformly bounded distortion. 
Let $G_{n}^{\prime} \subset M_{n}$ be such that $f^{k_{n}}\left(G_{n}^{\prime}\right)=G_{i_{n}}$. The uniformly bounded distortion of $F^{k_{n}} \mid M_{n}$ implies

$$
\frac{\left|G_{n}^{\prime}\right|}{\operatorname{dist}\left(y,\left|G_{n}^{\prime}\right|\right)} \geq \epsilon^{\prime}>0
$$

for some uniform $\epsilon^{\prime}>0$. Because $\left|T_{k_{n}}(y)\right| \rightarrow 0$ we get a contradiction.

】 (Lemma 3.2)

Let $V \supset U$ be the maximal interval which contains only the points $a$ and $b$ of the periodic orbits of $a$ and $b$. Clearly, for every $I \in \mathcal{I}$ there exists $E_{I} \supset I$ with $E_{I} \subset U$ such that

$$
f^{n_{I}}: E_{I} \rightarrow V
$$

is monotone onto.

Lemma 3.3. There exists $K>0$ such that

$$
\left|\left[(M \mid I)^{-1}\right]\right|_{2} \leq K
$$

for every $I \in \mathcal{I}$.

Proof: We have to show that for some $K>0$

$$
\left|\eta_{\left[(M \mid I)^{-1}\right]}\right|_{0} \leq K, I \in \mathcal{I} .
$$

Observe, that all the $[(M \mid I)]$ have, by construction, a monotone extension with negative Schwarzian derivative up to a fixed interval strictly bigger than $[-1,1]$. The Koebe-Lemma implies that for some $K>0$

$$
\ln \left(\frac{|D M(x)|}{|D M(y)|}\right) \leq K \cdot|x-y|,
$$

for any $x, y \in[-1,1]$. Hence,

$$
\left|\ln \left(\frac{\left[(M \mid I)^{-1}\right](x)}{\left[(M \mid I)^{-1}\right](y)}\right)\right| \leq K \cdot|x-y|,
$$

for any $x, y \in[-1,1]$. Consequently,

$$
\left|\int_{x}^{y} \eta_{\mid\left[(M \mid I)^{-1}\right]}\right|=\left|\ln \left(\frac{\left[(M \mid I)^{-1}\right](x)}{\left[(M \mid I)^{-1}\right](y)}\right)\right| \leq K \cdot|x-y|,
$$

for any $x, y \in[-1,1]$. This implies that $\left|\eta_{\left[(M \mid I)^{-1}\right]}\right|_{0} \leq K$.

【 (Lemma 3.3) The previous Lemmas allow us to construct, by rescaling range and domain of $M$, a $C^{2}$ Markov-map. The definition is essentially topological: we get a corresponding $C^{2}$ Markovmap on the domain $h(U)$ which is induced by $g$. The restriction $h \mid U$ of the multipliers preserving conjugacy $h$ between $f$ and $g$ becomes after rescaling a multiplier preserving 
conjugacy between the Markov-maps. Corollary 2.9 states that $h$ is a $C^{2}$ diffeomorphism. We proved that the conjugacy between $f$ and $g$ is $C^{2}$ when restricted to $\operatorname{int}\left(A_{f}\right)$.

Observe that $f\left(f^{2}(0)\right) \in \operatorname{int}\left(A_{f}\right)$. Now pull back the conjugation around $f\left(f^{2}(0)\right)$ two time to show that $h$ is also smooth in $f(0)$ and $f^{2}(0)$. We showed that $f$ and $g$ are smoothly conjugated on a neighborhood of their attractors.

Observe that in general these smooth conjugacy between the attractors can not be smoothly extended to the whole domain of the maps.

\section{Appendix}

The Schwarzian derivative of a $C^{3}$ map $f:[-1,1] \rightarrow[-1,1]$ is

$$
S f(x)=\frac{D^{3} f(x)}{D f(x)}-\frac{3}{2} \cdot\left(\frac{D^{2} f(x)}{D f(x)}\right)^{2} .
$$

where $D^{i} f(x)$ stands for the $i^{t h}$ derivative of $f$ in $x \in[-1,1]$.

Koebe-Distortion-Lemma. There exist continuous functions $K:(0, \infty) \rightarrow(0, \infty)$ and $\delta:(0, \infty) \rightarrow(0, \infty)$ with the following properties. Let $f^{n}: T \rightarrow f^{n}(T)$ be monotone and $S f(x) \leq 0$ for all $x \in[-1,1]$. If $I \subset T$ is an interval such that both component $L, R \subset T \backslash I$ satisfy

$$
\frac{\left|f^{n}(L)\right|}{\left|f^{n}(I)\right|}, \frac{\left|f^{n}(R)\right|}{\left|f^{n}(I)\right|} \geq \tau
$$

then $f^{n} \mid I$ has bounded distortion:

$$
\frac{\left|D f^{n}(x)\right|}{\left|D f^{n}(y)\right|} \leq 1+K(\tau) \frac{|x-y|}{|I|}
$$

for all $x, y \in I$ and

$$
\frac{|L|}{|I|}, \frac{|R|}{|I|} \geq \delta(\tau) .
$$

Moreover, $\lim _{\tau \rightarrow \infty} K(\tau)=1$ and $\lim _{\tau \rightarrow \infty} \delta(\tau)=\infty$.

A detailed discussion of the Koebe-Lemma can be found in [MS].

The Lebesgue measure on $[-1,1]$ is denoted by $|$.$| . A smooth map on the interval is called$ ergodic if the only measurable forward invariant sets have Lebesgue measure zero of full measure.

\section{References}

[BKNS] H.Bruin, G.Keller, T.Nowicki, S.van Strien, Wild Attractors Exist, Ann. of Math. 143 (1996), 
[BL] A.M.Blokh, M.Lyubich, Attractors of Maps on the interval, Func.Anal. and Appl. 21 (1987), 32-46.

[GMT] R.Galeeva, M.Martens, C.Tresser, Inducing, Slopes, and Conjugacy Classes, Israel Jour.Math. 99 (1997), 123-147.

[L1] M.Lyubich, Teichmüller Space of Fibonacci Maps, Stony Brook preprint 1993/12.

[L2] M.Lyubich, Combinatorics, Geometry and Attractors of Quasi-Quadratic Maps, Ann. of Math 140 (1994), 347-404.

[LM] M.Lyubich, J.W.Milnor, The Fibonacci Unimodal Map, J.A.M.S. 6 (1993), 425457.

[Ma] M.Martens, Distortion Results and Invariant Cantor Sets of Unimodal Maps, Erg.Th. \& Dyn.Sys. 14 (1994), 331-349.

[Mi] J.W.Milnor, On the Concept of Attractor, Commun. Math. Phys. 99 (1985), 177-195.

[MS] W.de Melo and S.van Strien, One-dimensional Dynamics, Springer-Verlag, 1993.

[SS] M.Shub, D.Sullivan Expanding Endomorphism of the Circle revisited, Erg.Th. \& Dyn.Sys. 5 (1985), 285-289. 